\title{
Evaluation of patellar tendon with shear wave elastography after anterior cruciate ligament reconstruction using hamstring tendons
}

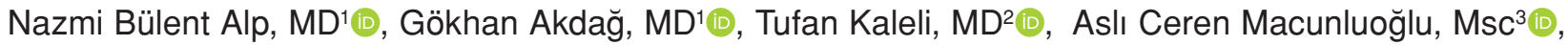 \\ Nail Uzunlulu, $\mathrm{MD}^{4}$ (D) \\ 1Department of Orthopedics and Traumatology, Istanbul Bahçelievler State Hospital, Istanbul, Turkey \\ ${ }^{2}$ Department of Orthopedics and Traumatology, Bursa Uludag University School of Medicine, Bursa, Turkey \\ ${ }^{3}$ Department of Biostatistics, Uludağ University, Institute of Health Sciences, Bursa, Turkey \\ ${ }^{4}$ Department of Radiology, Health Sciences University, Kanuni Sultan Süleyman Training and Research Hospital, Istanbul, Turkey
}

Ligament injuries are common injuries of the knee joint and the literature has reported that the most common one is the anterior cruciate ligament (ACL) injury. ${ }^{[1]}$ Anterior cruciate ligament ruptures are predicted to exist at a rate of 85 tears per 100,000 in individuals between the second and fourth decades. ${ }^{[1]}$ The ACL primarily prevents anterior translation of the tibia on the femur, and in addition to this, it also helps to balance the abnormal lateral rotation of the tibia. Anterior cruciate ligament deficiency accelerates many direct and long-term effects, including ache, muscle weakness, joint movement limitation, and in some cases, prolonged joint degeneration..$^{[2]}$

Patients who have suffered ACL rupture generally undergo an operation to reconstruct ACL with quadriceps, bone-patellar tendon-bone (BPTB), or hamstrings autografts. ${ }^{[3]}$ Among those tendons, hamstring tendons are widely used. The hamstring tendons consist of gracilis (GRA) and semitendinosus

Received: October 09, 2019

Accepted: October 24, 2019

Published online: March 02, 2020

Correspondence: Nazmi Bülent Alp, MD. Bursa Șehir Hastanesi Ortopedi ve Travmatoloji Kliniği, 16110 Doğanköy, Nilüfer, Bursa, Türkiye.

E-mail: nazmibulentalp@hotmail.com

Doi: $10.5606 /$ ehc. 2020.71745

Citation: Alp NB, Akdağ G, Macunluoğlu AC, Uzunlulu N, Kaleli T. Evaluation of patellar tendon with shear wave elastography after anterior cruciate ligament reconstruction using hamstring tendons. Jt Dis Relat Surg 2020;31(1):137-142.

\section{ABSTRACT}

Objectives: This study aims to evaluate the characteristics of patellar tendons (PTs) of patients who underwent anterior cruciate ligament reconstruction (ACLR) with hamstring tendon autograft using shear wave elastography (SWE).

Patients and methods: This prospective study was conducted between August 2017 and February 2018. We performed brightness mode (B-mode) ultrasound and SWE on 21 patients (20 males, 1 female; mean age 32 years; range, 19 to 42 years) who underwent ACLR with a single-bundle transtibial technique using quadruple hamstring autografts and 14 healthy controls (13 males, 1 female; mean age 35 years; range, 25 to 47 years). Length and thickness of the PT were evaluated with B-mode ultrasound, while elasticity values were evaluated with SWE in the patient and control groups.

Results: The mean SWE value of the patient group on the operated side was 25.30 ( $\min -\max =16.60-46.20)$ and on the non-operated side 23.20 ( $\min -\max =12.40-44)$. There were no statistically significant differences regarding PT elasticity, thickness, or length between the groups $(\mathrm{p}>0.05)$.

Conclusion: There were no differences regarding elasticity, length, or thickness between the operated and healthy knees detected with SWE. Upcoming research should focus on tendon biopsy and biochemical analyses for the identification of possible intrastructural changes of the tendon due to collagen synthesis after ACLR with hamstring autograft.

Keywords: Anterior cruciate ligament reconstruction, hamstring tendon, patellar tendon, shear wave elastography.

(ST) muscle groups. They are flexors and internal rotators of the knee joint. Hamstring strength is affected according to the use of an autologous ST/GRA tendon graft. ${ }^{[4]}$

The usage of autografts provides a higher return rate to the condition before the injury and a lessened chance of re-rupture than allografts; however, they 
may also cause morbidities, including short-term strength deficits in the donor site.

The patellofemoral pain syndrome may be seen in patients who undergo ACL reconstruction (ACLR). During the convalescence period, quadriceps muscle weakness occurs due to incomplete activation. ${ }^{[2]}$ This weakness within the quadriceps can take part in knee pain and simultaneously inhibit healing. ${ }^{[2]}$ Wang et al..$^{[5]}$ investigated the relationship of patellofemoral joint chondral lesions and strength of the quadriceps after ACLR with hamstrings. Their study reported that after ACLR, patients with quadriceps muscle strength greater than $80 \%$ were associated with less severe patellar cartilage damage in short-term follow-up. Their study evaluated not only pain levels but also cartilage injury, and provided additional evidence that, joint damage and anterior knee pain likely contibute to lasting quadriceps weakness. ${ }^{[2]}$ If the quadriceps tendon is one end of the rope, the other end is the patellar tendon (PT). Patellar tendon is a viscoelastic tendon, which contracts with quadriceps loading and takes a vital role in knee extension. ${ }^{[6-8]}$ We hypothesized that the weakness of the quadriceps muscle group attaching to the proximal part of the patella might affect the PT at the lower end.

As an alternative method, shear wave elastography (SWE) is used to approximate soft tissue elasticity ${ }^{[9]}$ In contradistinction to previous qualitative methods of ultrasound elastography, SWE is an ultrasoundbased technique that can describe the mechanical features of the tissue based on the spread of remotely induced shear waves. ${ }^{[10]}$ Shear wave elastography allows the direct measurement of elasticity in a wide zone within a previously defined region of interest (ROI). ${ }^{[1]}$ Recently, two small-scale studies revealed that SWE is a repeatable technique for the elasticity assessment of Achilles and PTs. ${ }^{[12,13]}$

Previous studies have investigated the PT healing or viscoelastic changes in patients who underwent ACLR using BPTB. However, as far as we know, there was no study in the literature evaluating the PT in patients who underwent ACLR using hamstring tendons. Therefore, in this study, we aimed to evaluate the characteristics of PTs of patients who underwent ACLR with hamstring tendon autograft using SWE.

\section{PATIENTS AND METHODS}

This prospective study was conducted at Istanbul Bahçelievler State Hospital, between August 2017 and February 2018. A total of 21 patients (20 males, 1 female; mean age 32 years; range, 19 to 42 years) who underwent ACLR with a single-bundle transtibial technique using quadruple hamstring autografts were included in the study. Subjects who underwent bilateral ACLRs, had a history of systemic disease, were aged younger than 18 years, underwent revision surgery, had previous fractures related to the knee joint, or multiple ligament injuries were excluded. The study protocol was approved by the University of Health Science, Bakırköy Dr. Sadi Konuk Training and Research Hospital Ethics Committee (No: 2017/160: 31-07-2017). A written informed consent was obtained from each participant. The study was conducted in accordance with the principles of the Declaration of Helsinki.

All patients underwent the same physiotherapy program, and SWE was performed three months after the ACLR. Fourteen healthy volunteers (13 males, 1 female; mean age 35 years; range, 25 to 47 years) were selected as the comparison group (height $=168.5 \pm 9.3 \mathrm{~cm}$, weight $=76.2 \pm 10.0$ ). Shear wave elastography and brightness mode (B-mode) ultrasound evaluations were performed by the same radiologist to eliminate inter-observer reliability. All ultrasound examinations were conducted using a Toshiba Aplio 500 ultrasound system (Toshiba Medical Systems Corporation, Tokyo, Japan) with a linear transducer $(12 \mathrm{MHz})$. Participants rested in lying position for five minutes before the examination. The ultrasound transducer head was aligned in the longitudinal plane by applying minimal pressure with the collagen fibers. The PT length was measured by measuring the distance from the lower end of the patella to the insertion of the tibial tuberosity (Figure 1), whereas elasticity and thickness (Figure 2) were measured by determining the distance between the central part of the proximal and distal PT attachments. The thickness and length of the PT were evaluated with the knee joint flexed at $15^{\circ}$, and elasticity of the tendon was evaluated when the knee

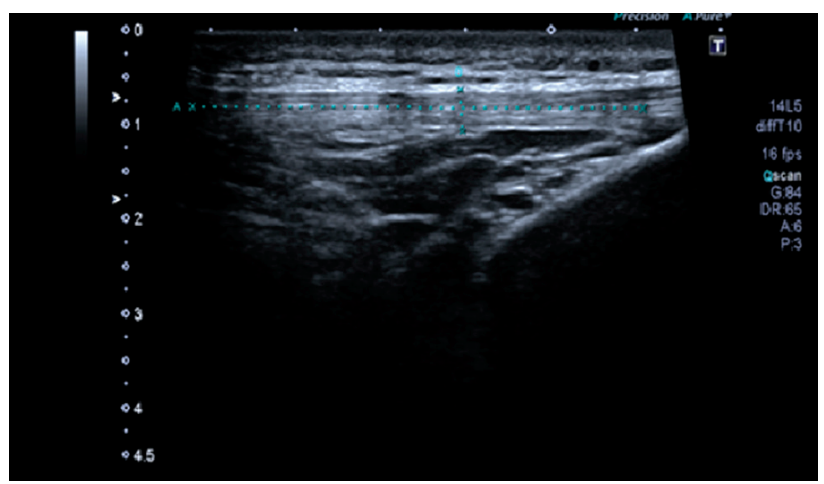

FIGURE 1. Patellar length measurement. 


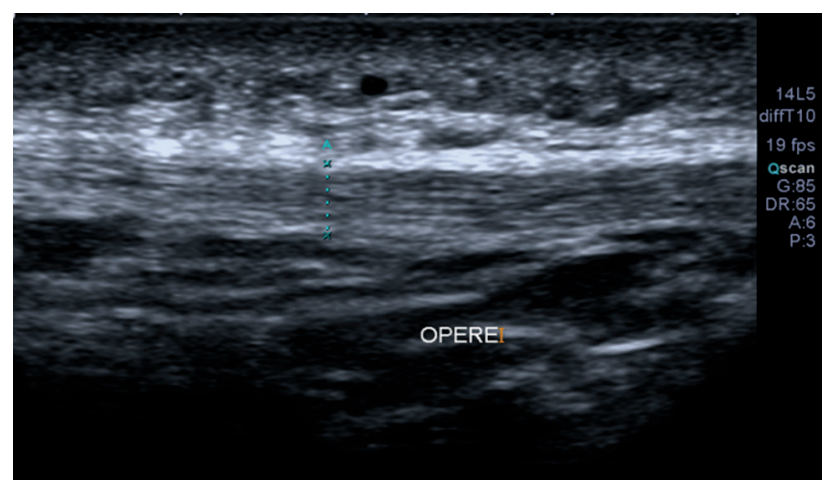

FIGURE 2. Patellar tendon thickness measurement.

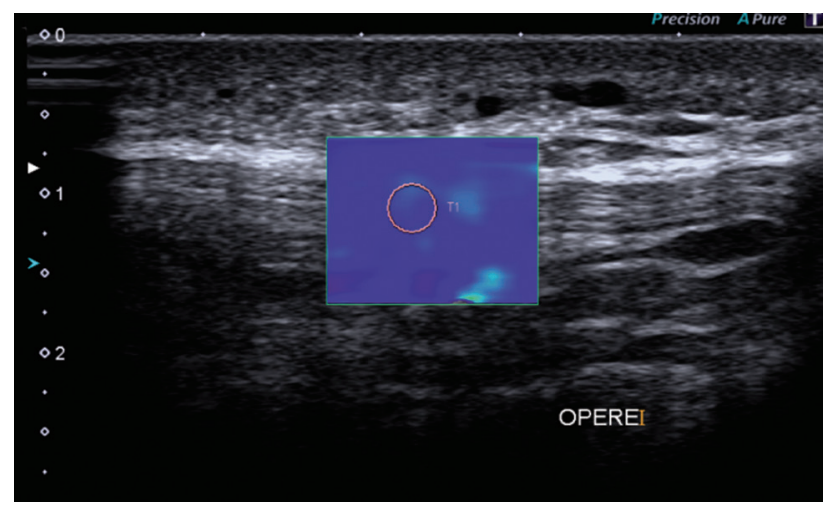

FIGURE 3. Region of interest measurement of patellar tendon elastography.

joint was in full extension position, appropriately to the method reported by Botanlioglu et al. ${ }^{[14]}$ Tendon length was measured in longitudinal real-time B-mode images using the in-built Toshiba distance function, and for elasticity measurements, SWE mode was switched on, and the transducer was held in the ROI approximately 10 seconds (Figure 3). Because of the potential disadvantages of SWE regarding adjacency to the bones, only the central part of the PT was evaluated for group comparison. Triplicate measurements were obtained for each location, and the mean values were utilized to analysis.

\section{Statistical analysis}

The prior power analysis was performed based on findings of the study conducted by Botanlioglu et al. ${ }^{[14]}$ The sample size calculation was based on mean \pm standard deviation values of the "elasticity" level. Elasticity level was found to be $74.1 \pm 24.7$ for the operated side and $47.7 \pm 15.3$ for the non-operated side for a total of 19 participants. The sample size for each group was computed as $n=11$ per group, and a total of 22 participants was estimated for a power

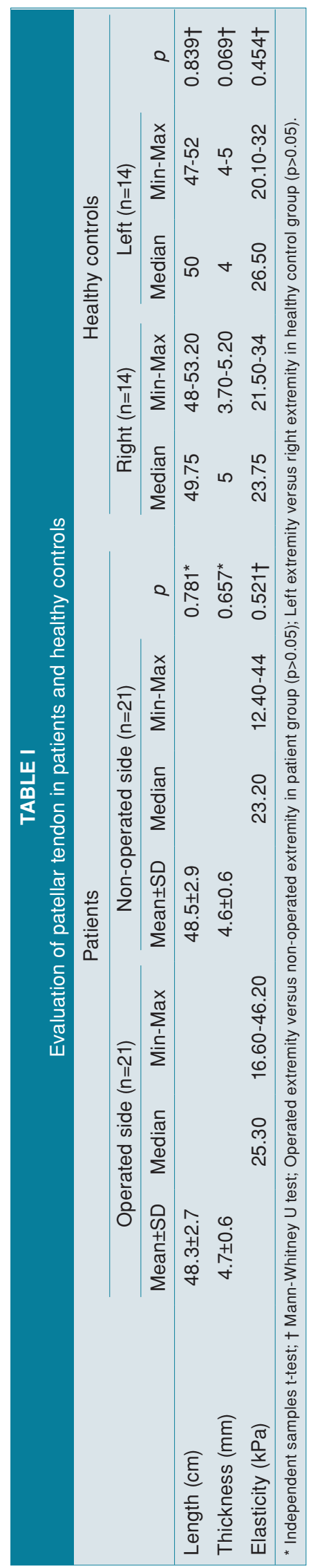


of 0.80 and alpha of 0.05 . (The moderate effect size $(\mathrm{d}=1.28)$. Power analysis was performed by using G*Power 3.1 (Heinrich-Heine-Universität Düsseldorf, Düsseldorf Germany). Shapiro Wilk test was used for the assessment of whether the data were normally distributed. Continuous variables were presented as median (min-max) and mean \pm standard deviation values. Independent samples t-test and Mann-Whitney $\mathrm{U}$ test were used for the comparison of two groups depending on the normality of the test result. The IBM SPSS version 20.0 (IBM Corp., Armonk, NY, USA) was used for performing the statistical analysis, and a $p$ value equal to or lower than 0.05 was considered statistically significant. Figures were generated by using GraphPad Prism version 7.0 (GraphPad Software Inc., San Diego, CA, USA).

\section{RESULTS}

There were no significant differences between the patient and control groups regarding gender or age $(p>0.05)$. In the patient group, the mean SWE value for the operated side was 25.30 (min-max=16.60-46.20) and for the non-operated side was 23.20 (min-max=12.40-44), while the mean SWE value in the control group for the right knee was 23.75 (min-max=21.50-34) and for the left knee was 26.50 ( $\min -\max =20.10-32$ ) (Table I). There were no statistically significant differences between the operated and non-operated sides, or between the right and left legs of the control group ( $p>0.05)$ (Table I).

\section{DISCUSSION}

Shear wave elastography technique used in the present study could not detect any significant difference in thickness, length, or elasticity of the PT of patients who had arthroscopic ACLR using quadruple GRA/ST autograft after an average of three-month follow-up.

The current literature has addressed that PT is susceptible to immobilization, aging, loading, and resistance exercise. Couppé et al. ${ }^{[15]}$ immobilized the knee joint at $30^{\circ}$ flexion for two weeks and investigated the effects over PT's mechanical properties in young and old male subjects. Their study results revealed that short-term immobilization had a negative effect on mechanical properties of the immobilized tendon side in both young and old males, and it could disrupt the function of the tendon-muscle complex. After the ACL repair, the whole body weight is superimposed on the non-operated side for some time, while no loadbearing is permitted on the operated knee during the early recovery period. In contrast to the mentioned study ${ }^{[15]}$ showing that short-term immobilization may disrupt the mechanical properties of the PT, our study did not detect any impairment over the mechanical properties of PT due to immobilization. In another study, Couppé et al. ${ }^{[16]}$ reported that cyclic loading was associated with an increase in PT's size and mechanical properties. Their findings revealed that the load extremity was $22 \%$ stronger than the nonload extremity, as well as having greater distal and proximal PT cross-sectional area; however, this was not clear at the mid-tendon level. Also, they showed that PT on the load extremity had higher stiffness (36\%) compared with the non-load extremity, whereas there was no significant difference in modulus. In another research, Kongsgaard et al. ${ }^{[17]}$ demonstrated that PT hypertrophy was observed in the distal and proximal parts but not in the middle of the tendon during resistance training.

On the other hand, Hsiao et al. ${ }^{[18]}$ found that SWE was a reproducible method for the evaluation of the elasticity of the PT with excellent intra- and interrater reliability in the middle area, but had good to fair reliability in the insertion area of both ends. Their study revealed that with aging, elastic modulus and shear wave velocity significantly decreased, and there were increased side-to-side differences compared with younger age groups. Because of the suboptimal reliability of SWE to adjacent bones, in the present study, the middle portion of the PT was used for the measurement of thickness and elasticity. After a three-month physiotherapy program, no significant differences were noted regarding the elasticity or thickness either in operated or healthy knees.

Moreover, Berko et al. ${ }^{[19]}$ assessed the effect of moderate exercise on the PT. Their study showed that PT softness was higher in young subjects and those engaging in moderate physical activity. Gulledge et al. ${ }^{[20]}$ performed an SWE study of healing PT following ACLR. Their findings suggested that there was an association between the mechanical properties of the PT and the post-surgery time in both affected and contralateral limbs after ACLR. Furthermore, Akkaya et al. ${ }^{[21]}$ reported elastography results of the PT in patients who underwent ACLR using patellar bone tendon-bone autograft. Their results revealed that PT on the operated sides seemed to thicken and shorten with decreased strain ratios at the donor side compared to the healthy side at an average of two years of follow-up. According to the last two studies performed, we think that the SWE measurements on the operated PT can only detect gross alterations.

There were several limitations associated with the present study. The most important one is 
that due to the suboptimal reliability of SWE to adjacent bones, only the middle portion of the PT was measured for the comparison of both groups. That suboptimal realibility may affected our study, thus the current disadvantages of the SWE method may have also been reflected in our study as well. We assumed that the studies in the literature have contradictory results regarding the measurements performed in the middle part of the PT, and randomized controlled trials are needed to justify the choice of a single ROI in the middle of the tendon being adequate to represent the characteristics of the entire tendon. In cross sectional studies as ours, it was impossible to certainly know whether or how elasticity changed over time in any of the patients. It may have changed over a certain time and then been equalized. Consequently, a longitudinal study is necessary to determine how elasticity changes over time. The small sample size was another limitation of our study.

In conclusion, improved knowledge on the collagen response of the human tendon against loading, unloading, strengthening, and overuse would guide us in exercise and rehabilitation regimens. There was no significant difference detected with the SWE method in the characteristics properties of the PT. Future studies should focus on tendon biopsy and biochemical analyses for the identification of possible changes in the internal structure of the tendon due to collagen synthesis. It is essential to clarify any difference in the collagen response of PT to ACLR with hamstring tendons.

\section{Declaration of conflicting interests}

The authors declared no conflicts of interest with respect to the authorship and/or publication of this article.

\section{Funding}

The authors received no financial support for the research and/or authorship of this article.

\section{REFERENCES}

1. Granan LP, Forssblad M, Lind M, Engebretsen L. The Scandinavian ACL registries 2004-2007: baseline epidemiology. Acta Orthop 2009;80:563-7.

2. Hubson A. The etiology of persistent quadriceps weakness following anterior cruciate ligament reconstruction. JBJS JOPA 2018;6:e24.

3. Akkaya S, Akkaya N, Güngör HR, Ağladıŏlu K, Ök N, Özçakar L. Sonoelastographic evaluation of the distal femoral cartilage in patients with anterior cruciate ligament reconstruction. Eklem Hastalik Cerrahisi 2016;27:2-8.

4. Suydam SM, Cortes DH, Axe MJ, Snyder-Mackler L, Buchanan TS. Semitendinosus Tendon for ACL Reconstruction: Regrowth and Mechanical Property Recovery. Orthop J Sports Med 2017;5:2325967117712944.
5. Wang HJ, Ao YF, Jiang D, Gong X, Wang YJ, Wang J, et al. Relationship between quadriceps strength and patellofemoral joint chondral lesions after anterior cruciate ligament reconstruction. Am J Sports Med 2015;43:2286-92.

6. Defrate LE, Nha KW, Papannagari R, Moses JM, Gill TJ, Li $\mathrm{G}$. The biomechanical function of the patellar tendon during in-vivo weight-bearing flexion. J Biomech 2007;40:1716-22.

7. Porta F, Damjanov N, Galluccio F, Iagnocco A, MatucciCerinic M. Ultrasound elastography is a reproducible and feasible tool for the evaluation of the patellar tendon in healthy subjects. Int J Rheum Dis 2014;17:762-6.

8. Ozcan AN, Tan S, Tangal NG, Cirac1 S, Kudas S, Bektaser SB, et al. Real-time sonoelastography of the patellar and quadriceps tendons: pattern description in professional athletes and healthy volunteers. Med Ultrason 2016;18:299-304

9. Bercoff J, Tanter M, Fink M. Supersonic shear imaging: a new technique for soft tissue elasticity mapping. IEEE Trans Ultrason Ferroelectr Freq Control 2004;51:396-409.

10. Eby SF, Song P, Chen S, Chen Q, Greenleaf JF, An KN. Validation of shear wave elastography in skeletal muscle. J Biomech 2013;46:2381-7.

11. Zhang ZJ, Ng GY, Lee WC, Fu SN. Changes in morphological and elastic properties of patellar tendon in athletes with unilateral patellar tendinopathy and their relationships with pain and functional disability. PLoS One 2014;9:e108337.

12. Chen XM, Cui LG, He P, Shen WW, Qian YJ, Wang JR. Shear wave elastographic characterization of normal and torn achilles tendons: a pilot study. J Ultrasound Med 2013;32:449-55.

13. Yurdaışık I. Comparison of two-dimensional shear wave elastography and point shear wave elastography techniques with magnetic resonance findings in detection of patellar tendinopathy. Eklem Hastalik Cerrahisi 2019;30:275-81.

14. Botanlioglu H, Kaynak G, Kantarci F, Guven MF, Zengin G, Aydingoz O. Length, thickness, and elasticity of the patellar tendon after closed wedge high tibial osteotomy: a shear wave elastographic study. J Orthop Surg (Hong Kong) 2016;24:194-7.

15. Couppé C, Suetta C, Kongsgaard M, Justesen L, Hvid LG, Aagaard P, et al. The effects of immobilization on the mechanical properties of the patellar tendon in younger and older men. Clin Biomech (Bristol, Avon) 2012;27:949-54.

16. Couppé C, Kongsgaard M, Aagaard P, Hansen P, BojsenMoller J, Kjaer M, et al. Habitual loading results in tendon hypertrophy and increased stiffness of the human patellar tendon. J Appl Physiol (1985) 2008;105:805-10.

17. Kongsgaard M, Reitelseder S, Pedersen TG, Holm L, Aagaard P, Kjaer M, et al. Region specific patellar tendon hypertrophy in humans following resistance training. Acta Physiol (Oxf) 2007;191:111-21.

18. Hsiao MY, Chen YC, Lin CY, Chen WS, Wang TG. Reduced patellar tendon elasticity with aging: In vivo assessment by shear wave elastography. Ultrasound Med Biol 2015;41:2899-905.

19. Berko NS, Hanstein R, Burton DA, Fornari ED, Schulz JF, Levin TL. Ultrasound elastography of the patellar tendon in young, asymptomatic sedentary and moderately active individuals. Clin Imaging 2019;54:172-7. 
20. Gulledge CM, Baumer TG, Juliano L, Sweeney M, McGinnis M, Sherwood A, et al. Shear wave elastography of the healing human patellar tendon following ACL reconstruction. Knee 2019;26:347-54.
21. Akkaya S, Akkaya N, Agladıoglu K, Gungor HR, Ok N, Özçakar L. Real-time elastography of patellar tendon in patients with auto-graft bone-tendon-bone anterior cruciate ligament reconstruction. Arch Orthop Trauma Surg 2016;136:837-42. 